\title{
Avoiding polyatomic interferences in measurements of lanthanides in uranium material for nuclear forensic purposes
}

\author{
Anna Vesterlund ${ }^{1,2}$ (D) Henrik Ramebäck ${ }^{1,2}$
}

Received: 28 April 2019 / Published online: 20 June 2019

(c) The Author(s) 2019

\begin{abstract}
Measurements of the lanthanide series with ICP-SF-MS provide low detection limits but suffer from oxides of the lighter lanthanides interfering on the heavier ones. In this work, two different methods to measure the lanthanide series without interferences, were investigated and compared to measuring the lanthanides directly with a standard sample introduction system. It is shown that by using a desolvating sample introduction system during measurements, the impact of polyatomic interferences are eliminated. It is also shown that using chemical separations to separate the elements in the lanthanide series into three fractions almost eliminates polyatomic interferences, while direct measurements with a standard sample introduction system may lead to inaccurate results due to interferences.
\end{abstract}

Keywords Nuclear forensics · Uranium · Lanthanides $\cdot$ ICP-MS

\section{Introduction}

Nuclear forensics is a scientific discipline that aims to aid in criminal investigations concerning illicit trafficking and use of nuclear material or other radioactive substances. The ultimate goal with an investigation is to find the origin and the intended use of the seized material. Nuclear forensics combine a number of methods to establish an attribution of nuclear or other radioactive material. Lanthanide patterns have proven to be a promising signature for determination of the geographical origin of uranium for nuclear forensic purposes [1-3]. The lanthanides are a series of rare elements which share similar chemical and physical properties and therefore maintain the same relative composition compared to each other even though the material undergo various chemical processes, such as uranium ore processing [4]. Another use for lanthanide patterns is material provenance in nuclear safeguards, where the aim is to confirm that the origin of declared nuclear material is consistent with the actual material [5].

Anna Vesterlund

anna.vesterlund@foi.se

1 CBRN Defence and Security, Swedish Defence Research Agency (FOI), Cementvägen 20, 90182 Umeå, Sweden

2 Department of Chemistry and Chemical Engineering, Nuclear Chemistry, Chalmers University of Technology, 41296 Göteborg, Sweden
The concentrations of the lanthanides are generally low in uranium material that has undergone various processes such as uranium ore processing to obtain nuclear fuel; therefore, a measurement technique with low detection limits, such as mass spectrometry, is needed. However, by using mass spectrometry, the concentrated uranium matrix may cause matrix effects resulting in decreased measurement sensitivity that, in turn, may increase the detection limits. The high amount of uranium introduced into the instrument may also cause memory effects, i.e. high uranium backgrounds that may be hard to eliminate. This might be an important factor if the same instrument is also used for uranium isotopic measurements. Varga et al. [6] proposed a method for group separation of the lanthanide series to remove uranium and barium from the samples using the TRU resin followed by lanthanide measurements using inductively coupled plasma-sector field-mass spectrometry (ICP-SF-MS). However, another problem with performing accurate lanthanide measurements by mass spectrometry is that some of the lanthanides, especially the lighter ones such as cerium and praseodymium, are prone to oxide formation in the plasma, causing polyatomic interferences at a higher mass [7]. This means that, for example, ${ }^{143} \mathrm{Nd}^{16} \mathrm{O}^{+}$will interfere with ${ }^{159} \mathrm{~Tb}^{+}$, which may lead to an overestimation of the amount of terbium. The higher the concentrations of the lighter lanthanides are compared to the heavier lanthanides, the larger the overestimation. 
In nuclear forensics, it is important that the accuracy and precision are maximized, in other words, it is important that the measurement uncertainties are well-understood and fit-for-purpose to make comparisons between materials useful $[8,9]$. Therefore, an overestimation of certain elements may be detrimental to the use of a measurement result. Attempts have been made to correct for these interferences mathematically $[10,11]$ but this approach may lead to large measurement uncertainties if the correction is large compared to the analyte in question and may require extensive measurements each day of analysis [12]. Funderberg et al. [13] presented a method for measuring the lanthanide series using medium-resolution LA-ICP-MS (laser ablation ICP-MS) which allows for peak deconvolution of the polyatomic interferences from the analytes. However, the method did not resolve the interference of ${ }^{143} \mathrm{Nd}^{16} \mathrm{O}^{+}$ on ${ }^{159} \mathrm{~Tb}^{+}$. The resolution needed to resolve these peaks is approx. 7700. Another downside with using medium or high mass resolution is that the sensitivity decreases significantly compared to low resolution and therefore results in higher detection limits [14]. Groopman et al. [15] presented the secondary ion mass spectrometry-single stage accelerator mass spectrometry (SIMS-SSAMS) as an excellent instrument for providing interference-free lanthanide patterns at low concentrations. However, SIMS-SSAMS is a rare technique and therefore there is a need for other, much more available, mass spectrometric techniques for low concentration element measurements. Inductively coupled plasma-mass spectrometry (ICP-MS) is a widespread, multi-elemental technique that is suitable for the purpose due to low detection limits for these elements. Even though many of the lanthanides have many isotopes, there is at least one isotope for each lanthanide free of isobaric interferences, but still the oxides, hydroxides and hydrides resulting in polyatomic interferences pose a problem.

The aim of this work was to examine the possibilities to measure the lanthanide series using ICP-SF-MS without interferences of oxides, hydroxides and hydrides. Two methods for minimizing the impact of oxides during measurements have been compared to conventional measurements without attempts to minimize the oxide impact on the results. The first method was to use a desolvating sample introduction system that dries the sample before it enters the plasma and therefore the oxide and hydride formation is kept at a minimum. The second method was to chemically separate the elements in the lanthanide series in such a way that the lighter elements are separated from the heavier elements during the measurement. A chemical separation method was developed for this purpose. The separation method also removes most of the uranium from the samples, making it possible to investigate the lanthanide pattern in materials with very low lanthanide concentrations, without risking contamination of the instrument with high amounts of uranium.

\section{Experimental}

All plasticware was acid-washed prior to use and all nitric acid used was in-house sub-boiled.

Reference materials REE-2 and CUP-2 (both CanmetMINING, Ottawa, Canada) were used for the study. REE-2 is a reference material certified for concentration of most lanthanides. CUP-2 is a uranium ore concentrate, which contains low, but uncertified amounts of lanthanides.

\section{Dissolution of reference materials}

CUP-2 was dissolved by microwave digestion (Mars 5, CEM Corporation, Matthews, US). $0.2 \mathrm{~g}$ was put in a Teflon tube. $9 \mathrm{~mL}$ concentrated $\mathrm{HNO}_{3}+0.09 \mathrm{~mol} \mathrm{~L}{ }^{-1} \mathrm{HF}$ (Suprapur, Merck KGaA, Darmstadt, Germany) was added together with $1 \mathrm{~mL}$ ultrapure water with $18.2 \mathrm{M} \Omega \mathrm{cm}$ resistivity at $25^{\circ} \mathrm{C}$ (Milli-Q, Merck KGaA, Darmstadt, Germany). The sample was digested by ramping the temperature to $180{ }^{\circ} \mathrm{C}$ for $20 \mathrm{~min}$ and holding at that temperature for $15 \mathrm{~min}$. After digestion, the sample was transferred to a bottle and the sample was diluted to a concentration of about $10 \mathrm{mg} \mathrm{U} \mathrm{g}^{-1}$ solution.

REE-2 was dissolved by lithium borate fusion due to incomplete dissolution using microwave digestion. $1 \mathrm{~g}$ of REE-2 was put in a carbon crucible together with $3 \mathrm{~g} \mathrm{LiBO}_{2}$ (Ultrapure, Claisse, Quebec, Canada). The sample was preoxidised for $2 \mathrm{~h}$ at $650{ }^{\circ} \mathrm{C}$ before fusing at $1050{ }^{\circ} \mathrm{C}$ for $15 \mathrm{~min}$. The fused sample was dissolved in $100 \mathrm{~mL} 10 \% \mathrm{HNO}_{3}$ while heating. After the sample had been dissolved, $0.4 \mathrm{~g}$ of polyethylene glycol (PEG-2000, Alfa Aesar, Karlsruhe, Germany) was added to flocculate silica and the solution was evaporated to approximately $50 \mathrm{~mL}$. The solution was left over night to let the slow silica flocculation progress. The solution was thereafter filtered through a filter paper with pore size 8-10 $\mu \mathrm{m}$ (Munktell OOM, Alstrom Munksjö, Helsinki, Finland) and diluted in $1 \mathrm{~mol} \mathrm{~L}^{-1} \mathrm{HNO}_{3}$. Blanks were prepared in the same way as CUP-2 and REE-2, respectively.

\section{Chemical separations}

For the separated samples, an aliquot was taken from the working solution. The sample was either diluted with Milli$\mathrm{Q}$ water to a concentration of $0.05 \mathrm{~mol} \mathrm{~L}^{-1} \mathrm{HNO}_{3}$ or evaporated and dissolved in $2 \mathrm{~mL} 0.05 \mathrm{~mol} \mathrm{~L}^{-1} \mathrm{HNO}_{3}$. For yield determination, a second sample was prepared for each sample by adding a known amount of a lanthanide multi-element solution. For the REE- 2 reference material, containing only low amounts of uranium, $1 \mathrm{mg}$ of uranium was added to each sample prior to the separation in order to mimic a uranium-rich material. Method blanks were prepared in the same manner. 
Two milliliter of Ln resin (di(2-ethylhexyl) orthophosphoric acid, HDEHP) was added to a $2 \mathrm{~mL}$ separation column (resin and separation column both from Triskem International, Bruz, France). The column was rinsed with $2 \times 2.5 \mathrm{~mL} 10 \mathrm{~mol} \mathrm{~L}^{-1} \mathrm{HNO}_{3}$ to remove possible lanthanides in the resin, followed by $2.5 \mathrm{~mL}$ Milli-Q water and $3 \times 2 \mathrm{~mL}$ $0.05 \mathrm{~mol} \mathrm{~L}^{-1} \mathrm{HNO}_{3}$. Another $2 \mathrm{~mL} 0.05 \mathrm{~mol} \mathrm{~L}^{-1} \mathrm{HNO}_{3}$ was added to the resin and the columns were sealed.

Prior to the separation, the columns were opened and allowed to drain. $1 \mathrm{~mL} 0.05 \mathrm{~mol} \mathrm{~L}^{-1} \mathrm{HNO}_{3}$ was added to the columns to condition the columns further. Thereafter the samples were added to the columns. The sample tubes were rinsed with $2 \times 0.5 \mathrm{~mL} 0.05 \mathrm{~mol} \mathrm{~L}^{-1} \mathrm{HNO}_{3}$ that were added to the columns. Lanthanum to neodymium were eluted with $6 \mathrm{~mL} 0.4 \mathrm{~mol} \mathrm{~L}^{-1} \mathrm{HCl}$, samarium to gadolinium were eluted with $10 \mathrm{~mL} 0.75 \mathrm{~mol} \mathrm{~L}^{-1} \mathrm{HCl}$ and terbium to lutetium were eluted with $20 \mathrm{~mL} 10 \mathrm{~mol} \mathrm{~L}^{-1} \mathrm{HNO}_{3}$ into Teflon beakers. All solutions were evaporated to near dryness and dissolved in $2 \% \mathrm{HNO}_{3}$ to change the solution to a more suitable matrix for ICP-MS measurements. The samples were, if needed, diluted to lanthanide concentrations less than approx. $2 \mathrm{ng} \mathrm{g}^{-1}$.

\section{Sample preparation}

For the direct measurements, the samples were diluted so that the concentration of the lanthanides in the measurement solution was kept between $6 \mathrm{pg} \mathrm{g}^{-1}$ and $2 \mathrm{ng} \mathrm{g}^{-1}$. An internal standard (indium, rhodium and rhenium) was added to all samples to a concentration of $1 \mathrm{ng} \mathrm{g}^{-1}$ of each element. In literature, all three elements have been chosen as internal standard for lanthanide measurements $[6,16,17]$. Initial experiments showed that the signal variation of each of the internal standards corresponded better with the signal variation of some of the lanthanides. Therefore, indium was used as internal standard for thulium, ytterbium and lutetium; rhenium was used as internal standard for terbium and dysprosium; and rhodium was used as internal standard for the rest of the lanthanide series.

\section{Measurements}

The measurements were performed on an Element2 (Thermo Fischer Scientific, Bremen, Germany). For the standard sample introduction a cyclonic spray chamber and concentric nebulizer were used (both GlassExpansion, Port Melbourne, Australia). For the measurements with a desolvating sample introduction system, a Cetac Aridus II (Teledyne Cetac Technologies, Omaha, Nebraska, US) together with a $100 \mu \mathrm{L} \mathrm{min}{ }^{-1} \mathrm{C}$-flow nebulizer was used. The instrumental settings and measurement parameters can be found in Tables 1 and 2. The instrument was tuned with a
Table 1 Instrumental settings for the two sample introduction systems

\begin{tabular}{|c|c|c|}
\hline \multirow[t]{2}{*}{ Instrumental settings } & Standard sample introduction & $\begin{array}{l}\text { Desolvating } \\
\text { sample intro- } \\
\text { duction }\end{array}$ \\
\hline & Twister spray chamber & Aridus II \\
\hline Nebulizer & Conikal & C-flow PFA \\
\hline Forward power [W] & 1250 & 1200 \\
\hline Cool gas flow [ $\left.\mathrm{L} \mathrm{min}^{-1}\right]$ & 16 & 16 \\
\hline Auxiliary gas flow $\left[\mathrm{L} \mathrm{min}^{-1}\right]$ & 0.7 & 0.7 \\
\hline Nebulizer gas flow $\left[\mathrm{L} \mathrm{min}^{-1}\right]$ & 1.1 & 0.9 \\
\hline Ar Sweep gas $\left[\mathrm{L} \mathrm{min}^{-1}\right]$ & N/A & 3.2 \\
\hline Nitrogen $\left[\mathrm{mL} \min ^{-1}\right]$ & N/A & 10 \\
\hline
\end{tabular}

Table 2 Measurement parameters

\begin{tabular}{ll}
\hline Measurement parameters & \\
\hline Resolution & 300 \\
Mass window & $5 \%$ \\
Samples per peak & 100 \\
Runs and passes & $100 \times 1$ \\
Scan type & $\mathrm{E}-\mathrm{scan}$ \\
Measured analyte isotopes & ${ }^{137} \mathrm{Ba},{ }^{139} \mathrm{La},{ }^{140} \mathrm{Ce},{ }^{141} \mathrm{Pr},{ }^{146} \mathrm{Nd},{ }^{147} \mathrm{Sm},{ }^{153} \mathrm{Eu}$, \\
& ${ }^{157} \mathrm{Gd},{ }^{159} \mathrm{~Tb},{ }^{163} \mathrm{Dy},{ }^{165} \mathrm{Ho},{ }^{167} \mathrm{Er},{ }^{169} \mathrm{Tm},{ }^{174} \mathrm{Yb}$, \\
Measured internal standard isotopes & ${ }^{103} \mathrm{Lu}$ \\
\hline
\end{tabular}


$1 \mathrm{ng} \mathrm{g}^{-1}$ cerium standard solution to minimize the oxide formation level of cerium while maintaining high sensitivity.

For the 5-point external calibration for the measurements of the unseparated samples, a multi-element standard (Sigma Aldrich, Buchs, Switzerland) was used. For the measurements of the separated samples, three different certified standard solutions were used, containing La-Nd, Sm-Gd and $\mathrm{Tb}-\mathrm{Lu}$, respectively (Spectrascan, Inorganic Ventures, Christiansburg, USA). For quality assurance, control samples were diluted from multi-element standards of another brand (CPAchem Ltd, Stara Zagora, Bulgaria). The multielement standards used for quality control had the same composition as the solutions used for calibration. All standard solutions used for calibration and quality assurance were certified by mass and traceable to NIST.

The dead-time was evaluated with the method proposed by Appelblad and Baxter [18] using a Lu standard solution. All data reduction and calculations were performed off-line. The external calibrations, using weighted linear regression, were carried out according to Sayago and Asuero [19] and the calculations as well as the measurement uncertainties

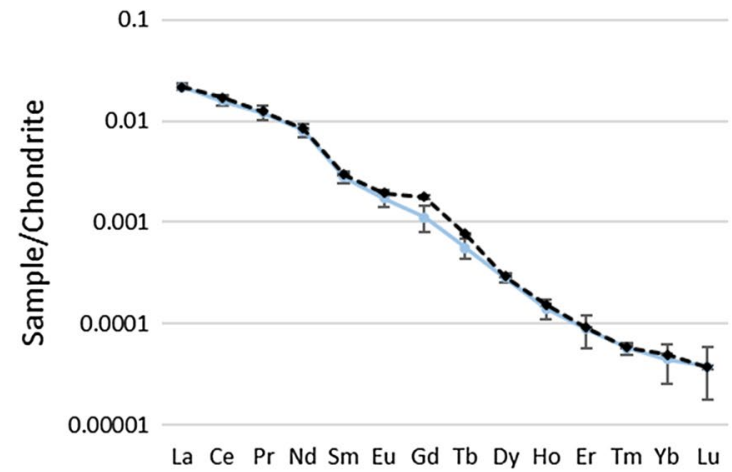

Fig. 1 Left: Comparison of the lanthanide pattern for REE-2 between certified values and results from direct measurement using a standard sample introduction system. Right: Relative deviation from the certi-

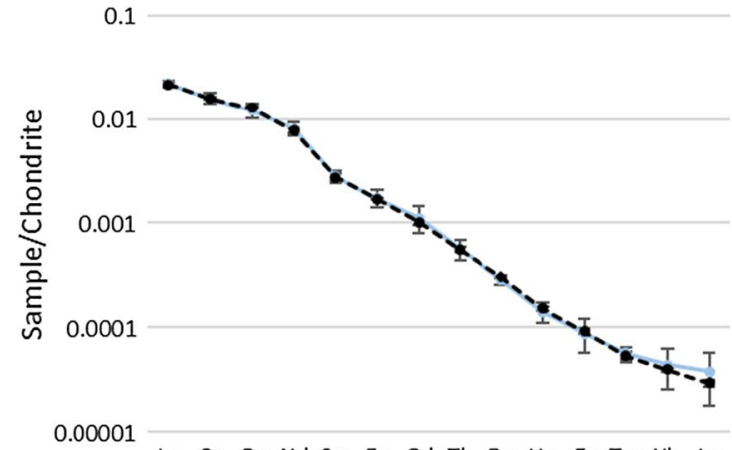

Fig. 2 Left: Comparison of the lanthanide pattern for REE-2 between certified values and measured results using a desolvating sample introduction system. Right: Relative deviation from the certified were evaluated using a Monte Carlo method in the same manner as Ramebäck and Lindgren [20].

The uncertainties were evaluated in accordance with ISO GUM [21]. All uncertainties are, unless stated otherwise, presented with a coverage factor $k=2$, corresponding to an approximate $95 \%$ confidence level. The measurement results were normalized using Chondrite values, see Figs. 1, 2, 3 and 4 [22].

\section{Results and discussion}

\section{Direct measurements}

The acquired pattern of the REE- 2 reference material from direct measurement using a standard sample introduction system can be seen in Fig. 1. For most elements, the results agree well with the certified values. The exceptions in this case are gadolinium and terbium, which are overestimated by approx. $60 \%$ and $40 \%$, respectively, see Table 3 . The highest amount of oxide formation can be seen in lanthanum,

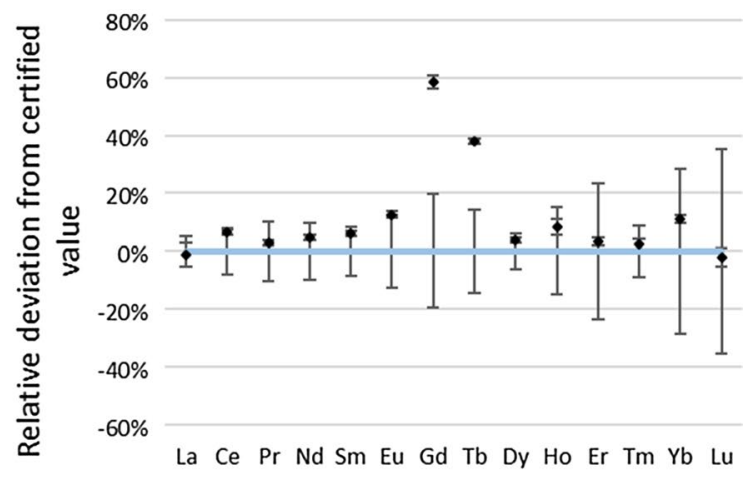

fied value. Diamonds are measured values. The continuous lines are the certified values. The uncertainty bars are, in some cases, smaller than the bullets

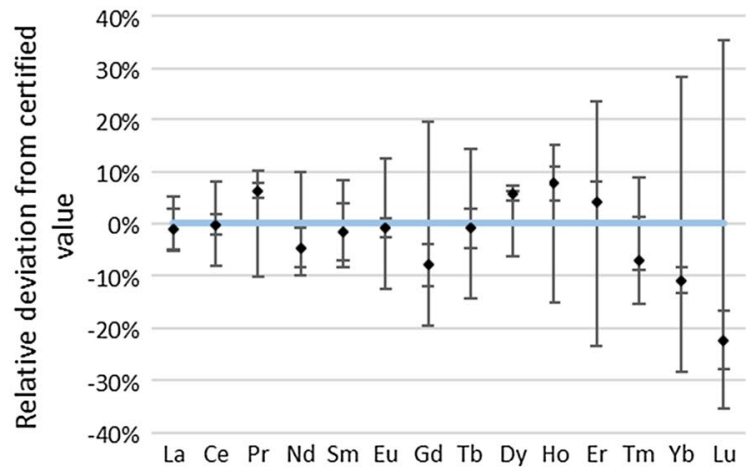

value. Diamonds are measured values. The continuous lines are the certified values. The uncertainty bars are, in some cases, smaller than the bullets 


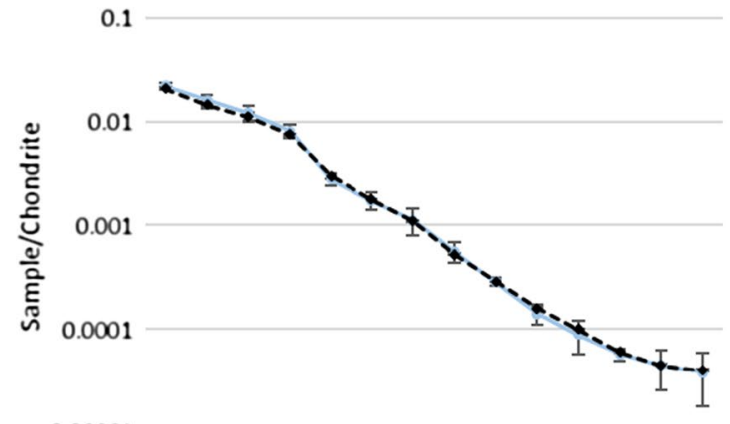

0.00001

La Ce Pr Nd Sm Eu Gd Tb Dy Ho Er Tm Yb Lu

Fig. 3 Left: Comparison of the lanthanide pattern for REE-2 between certified values and measured values using a standard sample introduction system after a chemical separation. Right: Relative deviation

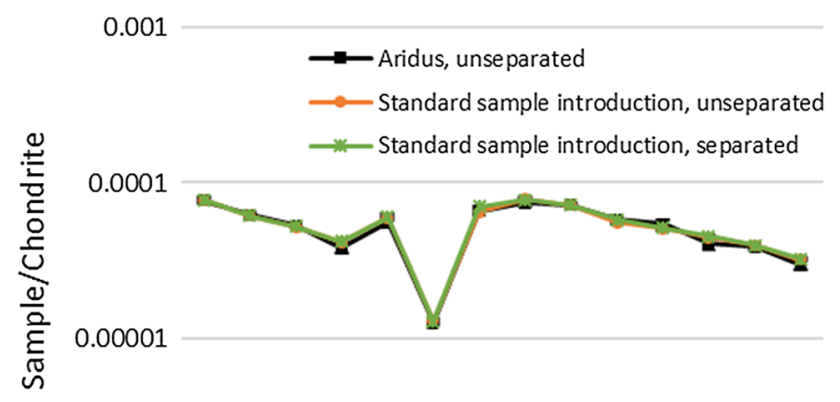

0.000001

La Ce Pr Nd Sm Eu Gd Tb Dy Ho Er Tm Yb Lu

Fig. 4 Comparison of the lanthanide pattern for CUP-2 between measurements using all three methods. The uncertainty bars are smaller than the bullets in most cases

cerium, praseodymium and neodymium. Oxides of praseodymium and neodymium will therefore interfere with masses 157 and 159 , for example. The $\mathrm{CeO} / \mathrm{Ce}$-ratio was approximately $2.5 \%$ at the time of measurement with the current measurement setup. The oxides of praseodymium and neodymium are expected to be lower but within the same order of magnitude [7, 23]. If the amounts of praseodymium and neodymium present in the sample are high enough compared to the amount of gadolinium and terbium, the oxides will start to interfere with the measurements of gadolinium and terbium.

Figure 2 shows the results of the direct measurements of REE-2 using a desolvating sample introduction system, compared to the certified values. At the time of measurement, the $\mathrm{CeO}$-formation was approx. 0.08\%. In this figure, it is clear that the interferences on gadolinium and terbium are removed; the results correspond well with the certified values.

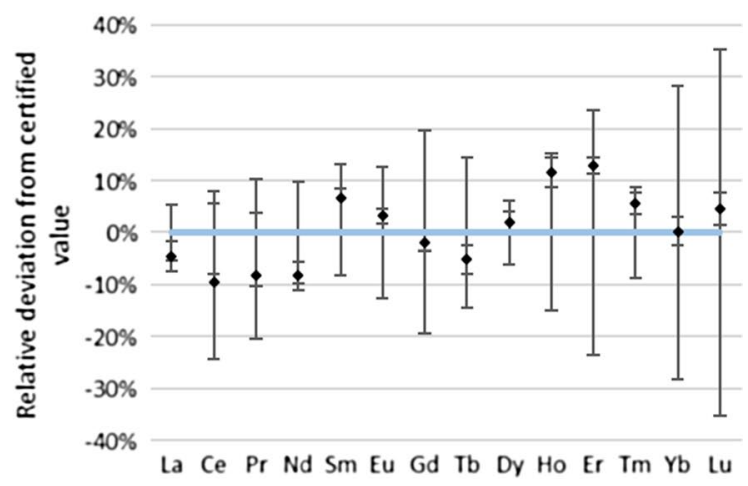

from the certified value. Diamonds are measured values. The continuous lines are the certified values. The uncertainty bars are smaller than the bullets in some cases

\section{Chemical separations}

The results of the measurements of REE- 2 after the chemical separation can be seen in Fig. 3. In this figure, it is evident that the interferences seen in Fig. 1 are absent. Since oxides of lighter lanthanides interfere with the heavier ones, e.g. ${ }^{141} \mathrm{Pr}^{16} \mathrm{O}^{+}$on ${ }^{157} \mathrm{Gd}^{+}$, it is preferable that $\mathrm{La}-\mathrm{Eu}$ and $\mathrm{Gd}-\mathrm{Lu}$ are measured in separate fractions. However, it has previously been shown that complete separation between $\mathrm{Eu}(\mathrm{III})$ and $\mathrm{Gd}(\mathrm{III})$ is difficult to perform in one single step using HDEHP [24-26]. Instead, a separation method including three fractions rather than two, was developed with the middle fraction containing $\mathrm{Sm}-\mathrm{Gd}$, which is more easily achieved. The separation method was developed from methods proposed previously using HDEHP as the extractant [27, 28]. By increasing the hydrochloric acid concentration, the lanthanides elute in groups. For the last elution, the acid was changed to nitric acid to avoid the elution of uranium, which will co-elute with the heavy lanthanides when high concentrations of hydrochloric acid are used [29, 30]. The mean chemical yield was $(100 \pm 2) \%(1 \mathrm{sd})$, which is similar to the yield achieved by Varga et al. [6] using TRU resin for lanthanide group separation. The range of the chemical yield was $95 \%$ to $107 \%$.

Other possible interferences are the oxides and hydrides from barium [7]. Performing a chemical separation of the lanthanide series using HDEHP as the extractant will also solve this problem since the $\mathrm{Ba}^{2+}$ ions are not extracted by HDEHP at the acid concentrations used in this work [31]. Using the desolvating sample introduction will also remove the barium hydrides and oxides in the same manner as for the lanthanides.

In Tables 3 and 4 it can be seen that the amount of the heavier elements are slightly lower when the results originate from the desolvating system compared to the standard sample introduction. This is due to interferences from the 
Table 3 Results and corresponding uncertainties from mass fraction measurements of REE-2

\begin{tabular}{|c|c|c|c|c|c|c|c|c|}
\hline & \multirow{2}{*}{\multicolumn{2}{|c|}{ Certified values }} & \multirow{2}{*}{\multicolumn{2}{|c|}{$\begin{array}{l}\text { Desolvating sample } \\
\text { introduction }\end{array}$}} & \multicolumn{4}{|c|}{ Standard sample introduction } \\
\hline & & & & & \multicolumn{2}{|c|}{ Direct measurement } & \multicolumn{2}{|c|}{ Separated samples } \\
\hline & $c\left[\mu \mathrm{g} \mathrm{g}^{-1}\right]$ & $U_{\mathrm{c}} k=2$ & $c\left[\mu \mathrm{g} \mathrm{g}^{-1}\right]$ & $U_{\mathrm{c}} k=2$ & $c\left[\mu \mathrm{g} \mathrm{g}^{-1}\right]$ & $U_{\mathrm{c}} k=2$ & $c\left[\mu \mathrm{g} \mathrm{g}^{-1}\right]$ & $U_{\mathrm{c}} k=2$ \\
\hline $\mathrm{La}$ & 5130 & 270 & 5080 & 200 & 5060 & 210 & 4890 & 140 \\
\hline $\mathrm{Ce}$ & 9610 & 770 & 9590 & 190 & 10,234 & 99 & 8700 & 1300 \\
\hline $\operatorname{Pr}$ & 1080 & 110 & 1148 & 17 & 1111 & 12 & 990 & 120 \\
\hline $\mathrm{Nd}$ & 3660 & 360 & 3490 & 130 & 3830 & 36 & 3356 & 92 \\
\hline $\mathrm{Sm}$ & 410 & 34 & 404 & 22 & 435.1 & 4.0 & 438 & 28 \\
\hline $\mathrm{Eu}$ & 97 & 12 & 95.8 & 1.7 & 108.7 & 1.3 & 99.6 & 1.4 \\
\hline Gd & $219^{1}$ & 43 & 201.7 & 8.1 & 346.9 & 8.1 & 214.6 & 3.4 \\
\hline $\mathrm{Tb}$ & 20.3 & 2.9 & 20.13 & 0.76 & 28.01 & 0.29 & 19.25 & 0.52 \\
\hline Dy & 69.2 & 4.3 & 73.2 & 1.1 & 71.69 & 0.64 & 70.5 & 1.6 \\
\hline Ho & 7.9 & 1.2 & 8.47 & 0.28 & 8.53 & 0.23 & 8.78 & 0.25 \\
\hline $\mathrm{Er}$ & 14.0 & 3.3 & 14.60 & 0.56 & 14.46 & 0.22 & 15.81 & 0.25 \\
\hline $\mathrm{Tm}$ & 1.38 & 0.12 & 1.29 & 0.11 & 1.415 & 0.026 & 1.461 & 0.030 \\
\hline $\mathrm{Yb}$ & $7.2^{1}$ & 2.0 & 6.41 & 0.16 & 8.00 & 0.12 & 7.21 & 0.20 \\
\hline $\mathrm{Lu}$ & $0.92^{1}$ & 0.33 & 0.715 & 0.040 & 0.899 & 0.030 & 0.962 & 0.030 \\
\hline
\end{tabular}

${ }^{1}$ Provisional value

\begin{tabular}{|c|c|c|c|c|c|c|}
\hline & \multirow{2}{*}{\multicolumn{2}{|c|}{ Desolvating sample introduction }} & \multicolumn{4}{|c|}{ Standard sample introduction } \\
\hline & & & \multicolumn{2}{|c|}{ Direct measurement } & \multicolumn{2}{|c|}{ Separated samples } \\
\hline & $c\left[\mu \mathrm{g} \mathrm{g}^{-1}\right]$ & $U_{\mathrm{c}} k=2$ & $c\left[\mu \mathrm{g} \mathrm{g}^{-1}\right]$ & $U_{\mathrm{c}} k=2$ & $c\left[\mu \mathrm{g} \mathrm{g}^{-1}\right]$ & $U_{\mathrm{c}} k=2$ \\
\hline $\mathrm{La}$ & 17.87 & 0.57 & 18.02 & 0.59 & 18.13 & 0.24 \\
\hline $\mathrm{Ce}$ & 37.27 & 0.63 & 36.81 & 0.41 & 36.80 & 0.72 \\
\hline $\operatorname{Pr}$ & 4.674 & 0.069 & 4.579 & 0.032 & 4.629 & 0.069 \\
\hline $\mathrm{Nd}$ & 16.97 & 0.91 & 18.56 & 0.21 & 18.67 & 0.35 \\
\hline $\mathrm{Sm}$ & 8.11 & 0.40 & 8.66 & 0.10 & 8.78 & 0.43 \\
\hline $\mathrm{Eu}$ & 0.701 & 0.014 & 0.723 & 0.014 & 0.711 & 0.016 \\
\hline Gd & 12.73 & 0.51 & 12.61 & 0.25 & 13.66 & 0.20 \\
\hline $\mathrm{Tb}$ & 2.68 & 0.10 & 2.832 & 0.035 & 2.80 & 0.11 \\
\hline Dy & 17.17 & 0.24 & 17.35 & 0.18 & 17.29 & 0.33 \\
\hline Ho & 3.20 & 0.11 & 3.066 & 0.076 & 3.177 & 0.080 \\
\hline $\mathrm{Er}$ & 8.52 & 0.31 & 8.02 & 0.10 & 8.05 & 0.31 \\
\hline $\mathrm{Tm}$ & 0.976 & 0.064 & 1.062 & 0.013 & 1.081 & 0.030 \\
\hline $\mathrm{Yb}$ & 6.20 & 0.15 & 6.376 & 0.078 & 6.41 & 0.12 \\
\hline $\mathrm{Lu}$ & 0.713 & 0.036 & 0.773 & 0.012 & 0.780 & 0.013 \\
\hline
\end{tabular}

Table 4 Results and corresponding uncertainties from the mass fraction measurements of CUP-2 to previously published results [16]. In the figure, all results are superimposed meaning that direct measurements with a standard introduction system are good enough to achieve the same results as with separated samples or the desolvating system. The reason for this is that the difference in concentrations between elements in the lanthanide series, in this material, are too small to result in amounts of oxides that would significantly alter the measured concentrations of the heavier lanthanides. However, direct measurements of uranium rich materials will cause memory effects in the instrument, which might be a problem if the same instrument has 
to be used for measuring uranium isotopics as well. Therefore, chemical separations might still be justified. In Table 4 it can be seen that the concentrations vary between approx. $40 \mu \mathrm{g} \mathrm{g}^{-1}$ (cerium) and $0.7 \mu \mathrm{g} \mathrm{g}^{-1}$ (europium and lutetium) in the CUP-2 material. For the REE-2 material, the concentrations vary between $10,000 \mu \mathrm{g} \mathrm{g}^{-1}$ (cerium) and $0.9 \mu \mathrm{g} \mathrm{g}^{-1}$ (lutetium), see Table 3.

\section{Measurement uncertainties}

In some cases, the results from the quality control samples did not correspond to the certified value. This discrepancy could not be explained in any other way than that there is a difference in concentration or underestimated uncertainty in the certified solutions used for calibration and quality control. Since it is difficult to determine which of the solutions that have the correct concentration this anomaly was addressed by adding an extra uncertainty component to the measurement model, corresponding to the uncertainty needed to force the control sample to correspond to the certified concentration of the control standard within their uncertainties:

$c_{\text {a.s }}=\left(\left(I_{\mathrm{a}}-a\right) / b\right)+\delta$

where $c_{\text {a.s }}$ is the calculated concentration of the analyte in the sample, $a$ and $b$ are intercept and slope, respectively, from the linear regression and $I_{\mathrm{a}}$ is the measured intensity of the analyte in the sample and $\delta$ is a constant with value 0 but with an uncertainty $u_{\mathrm{c}}(\delta)$. This approach ensures that the result of the measurement of the QC sample corresponds to the certified value within uncertainties at the $95 \%$ confidence level and has previously been applied on replicate samples by Kessel et al. [32] in a similar fashion.

The lowest uncertainties were achieved using direct measurement and the standard sample introduction system, since the signal stability was higher with the standard system than with the desolvating system. The highest uncertainties are calculated for the separated samples even though they are measured with the standard sample introduction system. The measurement uncertainty of the separated samples are on average around 3\% $(k=2)$ with a few exceptions where the uncertainty is somewhat higher. This is mainly due to the estimation of the uncertainty in the yield determination. This estimation was done by looking at the variation of the yields for each element and adding the $t$-factor corresponding to the degrees of freedom, to the calculated standard deviations. Since the number of yield determinations were small, this uncertainty contribution became significant. This explains, for example, the high uncertainty of cerium in Table 3. Other important uncertainty contributions are related to the calibration and/or the addition of the uncertainty of $\delta$ to account for discrepancies in the certified reference solutions. At very low concentrations, the uncertainty of the analyte signal contributes significantly to the combined uncertainty. The level of uncertainty, however, is, in general, at the same level or even below results presented by Varga et al. [6] and Asai and Limbeck [33].

The desolvating system also suffers more from matrix effects than the standard sample introduction. In the direct measurements, the concentration of uranium was $10 \mu \mathrm{g} \mathrm{g}^{-1}$. This concentration did not affect the sensitivity of the standard sample introduction to any extent, while the desolvating system suffered from an almost 50\% signal suppression. This was, however, to some extent, compensated by the higher sensitivity that can be achieved with a desolvating system.

It should be mentioned that the measurements in this study does not account for inhomogeneity in the CUP-2 material. The homogeneity of REE-2 is granted when more than $0.05 \mathrm{~g}$ of the material is used but in the case of CUP-2 there is no such information. In case of inhomogeneous material, multiple aliquots should be dissolved and measured and the variation between lanthanide mass fractions should be included in the uncertainty budget. This, of course, means that the measurement uncertainty would increase. It should also be noted that the combined uncertainties calculated from the certificate of the REE- 2 reference material are high. In some cases, the uncertainties are around $50 \%$. This reference material is also missing certified values for gadolinium, ytterbium and lutetium. The values used in those cases are provisional values. Therefore, this reference material may be unsuitable as a reference material for nuclear forensic purposes if these elements have to be measured, but is a good example of when direct measurements with a standard sample introduction system are inappropriate.

\section{Conclusions}

This work has shown that it is possible to measure the lanthanide series with good precision and accuracy independent of the lanthanide pattern profile. Two methods have been tested with satisfying results: direct measurement of a uranium solution using a desolvating sample introduction system and measurement of separated samples using standard sample introduction. This was compared with direct measurement of a uranium solution and a standard sample introduction system. The results show that direct measurements using the standard method may result in a substantial bias in the concentration for certain elements due to oxide formation in the plasma. This means that if a laboratory has access to a desolvating sample introduction system there is a quick and easy way to obtain, essentially, interferencefree lanthanide measurement data. Another advantage of the 
desolvating sample introduction system is that the relative sensitivity, in general, increases compared to the normal sample introduction system.

If there is no access to a desolvating system, the other possibility to achieve interference-free measurements of the lanthanide series is to perform a chemical separation on the material to separate interfering from interfered elements and in that way avoid the oxide interferences. Another approach is to combine the desolvating sample introduction with chemical separation to remove the concentrated uranium matrix which otherwise may cause severe signal suppression in the dry plasma. This combination could provide interference-free, high sensitivity measurements of materials containing very low amounts of lanthanides.

Acknowledgements The Swedish Civil Contingencies Agency, Project No. B40095, is gratefully acknowledged for funding this work.

Open Access This article is distributed under the terms of the Creative Commons Attribution 4.0 International License (http://creativeco mmons.org/licenses/by/4.0/), which permits unrestricted use, distribution, and reproduction in any medium, provided you give appropriate credit to the original author(s) and the source, provide a link to the Creative Commons license, and indicate if changes were made.

\section{References}

1. Mercadier J, Cuney M, Lach P, Boiron M-C, Bonhoure J, Richard A, Leisen M, Kister P (2011) Origin of uranium deposits revealed by their rare earth element signature. Terra Nova 23:264-269

2. Spano TL, Simonetti A, Balboni E, Dorais C, Burns PC (2017) Trace element and $U$ isotope analysis of uraninite and ore concentrate: applications for nuclear forensic investigations. Appl Geochem 84:277-285

3. Varga Z, Wallenius M, Mayer K (2010) Origin assessment of uranium ore concentrates based on their rare-earth elemental impurity pattern. Radiochim Acta 98:771-778

4. Varga Z, Krajko J, Peńkin M, Novák M, Eke Z, Wallenius M, Mayer K (2017) Identification of uranium signatures relevant for nuclear safeguards and forensics. J Radioanal Nucl Chem 312:639-654

5. Peńkin M, Boulyga S, Fischer D (2016) Application of uranium impurity data for material characterization in nuclear safeguards. J Radioanal Nucl Chem 307:1995-1999

6. Varga Z, Katona R, Stefánka Z, Wallenius M, Mayer K, Nicholl A (2010) Determination of rare-earth elements in uranium-bearing materials by inductively coupled plasma mass spectrometry. Talanta 80:1744-1749

7. Dulski P (1994) Interferences of oxide, hydroxide and chloride analyte species in the determination of rare earth elements in geological samples by inductively coupled plasma-mass spectrometry. Fresenius J Anal Chem 350:194-203

8. Leggitt J, Inn K, Goldberg S, Essex R, LaMont S, Chase S (2009) Nuclear forensics-metrological basis for legal defensibility. J Radioanal Nucl Chem 282:997-1001

9. Nuclear Forensics Support (2006) IAEA Nuclear Security Series No. 2: Technical Guidance, Vienna
10. Raut NM, Huang L-S, Aggarwal SK, Lin K-C (2003) Determination of lanthanides in rock samples by inductively coupled plasma mass spectrometry using thorium as oxide and hydroxide correction standard. Spectrochim Acta 58B:809-822

11. Vaughan M, Horlick G (1990) Correction procedures for rare earth element analyses in inductively coupled plasma-mass spectrometry. Appl Spectrosc 44:587-593

12. Simitchiev K, Stefanova V, Kmetov V, Andreev G, Sanchez A, Canals A (2008) Investigation of ICP-MS spectral interferences in the determination of $\mathrm{Rh}, \mathrm{Pd}$, and $\mathrm{Pt}$ in road dust: assessment of correction algorithms via uncertainty budget analysis and interference alleviation by preliminary acid leaching. Talanta 77:889-896

13. Funderberg R, Arevalo Jr R, Locmelis M, Adachi T (2017) Improved precision and accuracy of quantification of rare earth element abundances via medium-resolution LA-ICP-MS. J Am Soc Mass Spectrom 28:2344-2351

14. Nelms S (2005) ICP mass spectrometry handbook. Blackwell publishing Ltd, Oxford

15. Groopman E, Grabowski K, Fahey A, Kööp L (2017) Rapid, molecule-free, in situ rare earth element abundances by SIMSSSAMS. J Anal At Spectrom 32:2153-2163

16. Balboni E, Simonetti A, Spano T, Cook N, Burns P (2017) Rareearth element fractionation in uranium ore and its $\mathrm{U}(\mathrm{VI})$ alteration minerals. Appl Geochem 87:84-92

17. Lawrence MG, Greig A, Collerson KD, Kamber BS (2006) Direct quantification of rare earth element concentrations in natural waters by ICP-MS. Appl Geochem 21:839-848

18. Appelblad P, Baxter D (2000) A model for calculating deadtime and mass discrimination correction factors from inductively coupled plasma mass spectrometry calibration curves. J Anal At Spectrom 15:557-560

19. Sayago A, Asuero A (2004) Fitting straight lines with replicated observations by linear regression: part II. Testing for Homogeneities of Variances. Crit Rev Anal Chem 34:133-146

20. Ramebäck H, Lindgren $P$ (2018) Uncertainty evaluation in gamma spectrometric measurements: uncertainty propagation versus Monte Carlo simulation. Appl Radiat Isot 142:71-76

21. ISO: Guide to the Expression of Uncertainty in Measurement (1995) International Organisation for Standardisation, Geneva, Switzerland

22. Anders E, Grevesse N (1989) Abundances of the elements: meteoritic and solar. Geochim Cosmochim Acta 53:197-214

23. Longerich H, Fryer B, Strong D, Kantipuly C (1987) Effects of operating conditions on the determination of the rare earth elements by inductively coupled plasma-mass spectrometry (ICPMS). Spectrochim Acta 42B:75-92

24. Nash KL, Jensen MP (2000) Analytical separations of the lanthanides: basic chemistry and methods. In: Gschneidner KA, Eyring L (eds) Handbook on the physics and chemistry of rare earths, vol 28. Elsevier Science B.V., North-Holland

25. Morais CA, Ciminelli VST (1998) Recovery of europium from a rare earth chloride solution. Hydrometallurgy 49:167-177

26. Morais CA, Ciminelli VST (2007) Selection of solvent extraction reagent for the separation of europium(III) and gadolinium(III). Miner Eng 20:747-752

27. Pin C, Zalduegui JFS (1997) Sequential separation of light rareearth elements, thorium and uranium by miniaturized extraction chromatography: application to isotopic analyses of silicate rocks. Anal Chim Acta 339:79-89

28. Yang Y, Zhang H, Chu Z, Xie L, Wu F (2010) Combined chemical separation of $\mathrm{Lu}, \mathrm{Hf}, \mathrm{Rb}, \mathrm{Sr}, \mathrm{Sm}$ and $\mathrm{Nd}$ from a single rock digest and precise and accurate isotope determinations of $\mathrm{Lu}-\mathrm{Hf}, \mathrm{Rb}-\mathrm{Sr}$ and $\mathrm{Sm}-\mathrm{Nd}$ isotope systems using multi-collector ICP-MS and TIMS. Int J Mass Spectrom 290:120-126 
29. Shabana R, Ruf H (1977) Extraction and separation of uranium, thorium and cerium from different mixed media with HDEHP. J Radioanal Chem 36:389-397

30. Kaminski M, Nuñez L (2000) Separation of uranium from nitricand hydrochloric-acid solutions with extractant-coated magnetic microparticles. Sep Sci Technol 35:2003-2018

31. Horwitz E, Bloomquist C (1975) Chemical separations for superheavy element searches in irradiated uranium targets. J Inorg Nucl Chem 37:425-434

32. Kessel R, Berglund M, Wellum R (2008) Application of consistency checking to evaluation of uncertainty in multiple replicate measurements. Accred Qual Assur 13:293-298
33. Asai S, Limbeck A (2015) LA-ICP-MS of rare earth elements concentrated in cation-exchange resin particles for origin attribution of uranium ore concentrate. Talanta 135:41-49

Publisher's Note Springer Nature remains neutral with regard to jurisdictional claims in published maps and institutional affiliations. 